
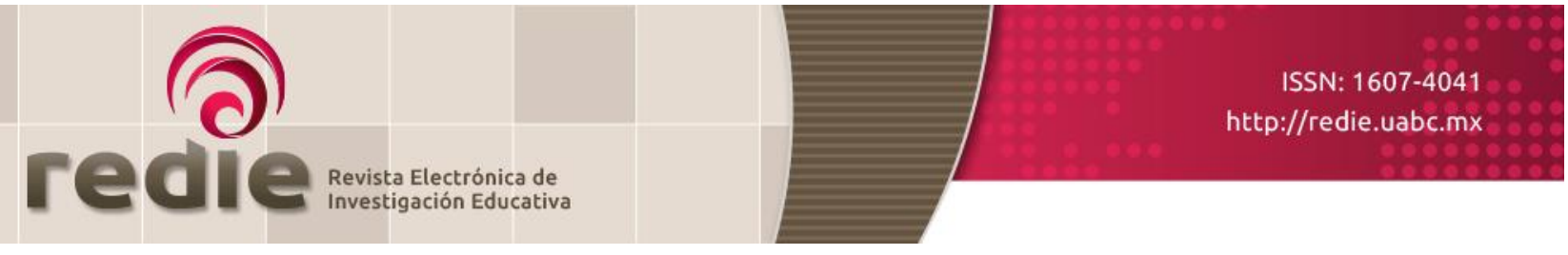

Vol. 22, 2020/e13

\title{
Representaciones sociales de docentes sobre la inclusión del estudiantado con discapacidad
}

\section{Teachers' Social Representations Concerning the Inclusion of Students with Disabilities}

Mario Fernando Gutiérrez (1) https://orcid.org/0000-0003-3452-8658

Liliana Martínez Fernández (2) https://orcid.org/0000-0002-1654-2395

(1) Pontificia Universidad Javeriana

(2) Universidad del Valle

(Recibido: 14 de febrero de 2018; Aceptado para su publicación: 31 de mayo de 2018)

Cómo citar: Gutiérrez, M. F. y Martínez, L. (2020). Representaciones sociales de docentes sobre la inclusión del estudiantado con discapacidad. Revista Electrónica de Investigación Educativa, 22, e13, 1-13. https://doi.org/10.24320/redie.2020.22.e13.2260

\section{Resumen}

El artículo busca identificar las representaciones sociales que poseen los docentes de instituciones educativas públicas de Bogotá y Cali (Colombia) sobre la inclusión de niños con discapacidad en las aulas. La investigación contempló el uso de análisis inductivos propios de la teoría fundamentada, para ello se identificaron los contenidos y la estructura interna de las representaciones sociales (análisis descriptivo y relacional). En el estudio participaron 25 docentes de dos escuelas públicas de Bogotá y Cali. Los resultados evidencian que los docentes consideran que, en la inclusión escolar, ellos juegan un rol articulador entre las políticas públicas y la implementación pedagógica en el aula, aunque no siempre con la preparación adecuada. Para los docentes el aspecto emocional de la experiencia inclusiva es demandante y lo valoran mayoritariamente-como negativo.

Palabras clave: Educación especial, integración escolar, necesidades educativas, derecho a la educación.

\section{Abstract}

This article seeks to identify the social representations held by public school teachers in Bogotá and Cali (Colombia) regarding the inclusion of disabled children in the classroom. The research followed an inductive approach based on grounded theory; to this effect, the content and internal structure of social representations were identified (a descriptive and relational analysis). The study involved 25 teachers from two public schools in Bogotá and Cali. The results show that, as far as inclusion is concerned, teachers believe they play a coordinating role between public policy and pedagogical implementation in the classroom, despite not always having appropriate training. For teachers, the emotional dimension of inclusion is demanding, and - for the most part - this is viewed negatively.

Keywords. Special education, pupil integration, educational needs, right to education. 


\section{Introducción}

Más de 20 años han pasado desde que la conferencia mundial sobre educación y necesidades educativas especiales, realizada en Salamanca (España), pusiera de manifiesto la necesidad de que todos los niños, sin importar su condición, por razones de raza, sexo, religión o necesidades educativas particulares, asistieran a la escuela regular y gozaran de las mismas oportunidades de aprendizaje que el resto de los niños de su edad (UNESCO, 1994). Desde entonces, hablar de educación inclusiva hace referencia a la búsqueda permanente de formas adecuadas de responder a la diversidad de los estudiantes (bajo los principios éticos de justicia social, equidad y responsabilidad), en la que se respeten las singularidades, se creen contextos pertinentes a las necesidades individuales y se promueva la participación social.

Ainscow et al. (2013) señalan que el concepto de equidad, subyacente a la educación inclusiva, permite comprender que las experiencias y resultados de los estudiantes no dependen sólo de las prácticas educativas de sus profesores, ni siquiera de sus escuelas, sino que vienen determinados por un amplio abanico de procesos interactivos que llegan a la escuela desde el exterior -como la demografía urbana, las historias y culturas de las poblaciones que escolarizan (o no) a los infantes- y las realidades económicas a las que se enfrentan estas poblaciones, entre otros.

El objetivo de la educación inclusiva es que todos los niños reciban y se beneficien de los apoyos que necesitan para desarrollar sus potencialidades sin tener en cuenta sus características personales por cuestiones de raza, religión, género o capacidades. En esta línea de ideas, el desafío de la escuela es transformarse al máximo para ofrecer a los niños la posibilidad de acceder a los aprendizajes, respetando sus características y facilitando su participación en todas las actividades escolares. Esto se traduce en modificaciones profundas al sistema educativo que permitan avanzar en la formulación de propuestas educativas flexibles que tengan en cuenta al otro, reconociendo sus capacidades y potencialidades (Ministerio de Educación Nacional de Colombia [MEN], 2012).

Ainscow (2012) afirma que para lograr que el aula regular se transforme y dé respuesta a las necesidades de todos los alumnos se requiere que se cuestionen las repercusiones de los roles de las personas que se desempeñan en el ámbito de la educación especial y el apoyo pedagógico. Este artículo busca contribuir a la comprensión de aspectos interpersonales que afectan a los docentes en su práctica cotidiana.

La discapacidad, entendida como un fenómeno complejo que refleja una interacción entre las características del ser humano y las de la sociedad en la que vive, es uno de los temas que genera mayor inquietud cuando se piensa en los procesos de inclusión educativa. Atender la población de niños y adolescentes que tienen una condición de discapacidad no ha sido un proceso sencillo, menos aun cuando los registros históricos muestran las distintas concepciones que sobre la discapacidad se han tenido a través del tiempo (Yarza, 2010; Yarza et al., 2013).

Uno de los aspectos que resalta la literatura en torno al trabajo que la escuela debe llevar a cabo está relacionado con las representaciones sociales que tienen los profesores hacia los niños en situación de discapacidad (Garnique-Castro y Gutiérrez-Vidrio, 2012). Las representaciones sociales pueden ser definidas según Moscovici (1961) como una modalidad del conocimiento, cuya función es la elaboración de los comportamientos y la comunicación entre los individuos.

La representación sería un corpus organizado de conocimientos gracias al cual se haría inteligible la realidad física y social (Moscovici, 1961). Para Jodelet (1986; 2006) las representaciones sociales (RS) constituyen maneras de interpretar y de pensar la realidad cotidiana, formas de conocimiento sobre el mundo, implicando en la definición las operaciones mentales que los individuos realizan en dichos contextos de cotidianidad. Las RS pueden ocasionar diversas respuestas por parte de docentes hacia los niños en situación de discapacidad que van desde una interacción positiva debido a una propuesta pedagógica adaptada a sus necesidades, hasta la falta de oportunidades similares de aprendizaje para éstos, debido a la carencia de criterios equitativos (Hevia, 2010; como se citó en Brito y Mansilla, 2013). 
Estas representaciones pueden ser también un predictor de las conductas y emociones de los docentes hacia los estudiantes en situación de discapacidad (Cuevas, 2015; Thaver et al., 2014), y cuya identificación debe ser explícita en los programas de formación docente, pues influyen en la forma en que se acepta ser educador de estudiantes en situación de discapacidad y, por consiguiente, en su identidad profesional (Hassan et al., 2015). Garnique-Castro y Gutiérrez-Vidrio (2012) han analizado las RS de docentes en México sobre la incorporación de la inclusión en las aulas escolares permitiendo identificar que dichas representaciones guían sus actitudes, valores y comportamientos dentro del aula.

En el presente artículo se propone como problema de investigación establecer cuáles son las Rs que tienen docentes de educación oficial en dos ciudades de Colombia (Bogotá y Cali) sobre el proceso de inclusión de niños con discapacidad. Los objetivos fueron identificar las RS de los docentes sobre las exigencias que conlleva la implementación cotidiana de la educación inclusiva e indagar el orden jerárquico de la dificultad que los docentes les atribuyen, dentro de sus RS, al conocimiento previo sobre discapacidad y a la emoción suscitada. Se espera contribuir así a la comprensión de algunos de los aspectos que subyacen al fenómeno de la inclusión educativa en escuelas públicas en Colombia y que se vuelven determinantes para su viabilidad práctica.

\section{Método}

La investigación se sitúa en el contexto de las RS frente a la escolarización de niños en situación de discapacidad. En total fueron entrevistados 25 profesores de aula regular del nivel de básica primaria (18 mujeres y 7 hombres), de distintas instituciones educativas públicas de Bogotá y Cali, con al menos 10 años de experiencia docente. Los profesores cumplían con el criterio de tener experiencia ${ }^{1}$ en la educación de niños con discapacidad y querer participar de forma voluntaria en la investigación. Todos dieron a conocer su intención de participar a través de la firma de un consentimiento informado. La muestra fue, por tanto, intencional y no probabilística.

Los docentes fueron entrevistados en las instalaciones donde laboran, en espacios donde se garantizaba su privacidad y sin la imposición de un límite de tiempo, de tal forma que tenían un nivel de privacidad suficiente para responder a la entrevista sin presiones, ni ruidos, ni coacciones por parte de los directivos de las instituciones en las que trabajaban. Las entrevistas fueron transcritas y posteriormente analizadas.

Se optó por una entrevista semiestructurada orientada por una matriz de preguntas agrupadas en tres dimensiones: 1) información que tienen los docentes en relación con los conceptos de discapacidad e inclusión escolar; 2) las emociones que se desprenden de su quehacer docente, y 3) el quehacer diario del docente y los hechos que favorecen o dificultan la ejecución de los procesos inclusivos en el aula; esta dimensión hace referencia al orden jerárquico de los elementos que contiene una representación (España y Gentiletti, 2011).

Para identificar las RS se acude al concepto de Teoría Fundamentada (TF) que, siendo un enfoque dentro de la metodología cualitativa, permite crear teorías basándose exclusivamente en los datos (Campo-Redondo y Labarca, 2009). En la TF los datos son la base de la teoría emergente y éstos pueden provenir de entrevistas y observaciones, entre otros documentos (Strauss y Corbin, 2002). Del manejo conjunto de los datos entre los investigadores y los datos surgen las categorías con sus propiedades y dimensiones, elementos fundamentales para el desarrollo de la teoría. Las "categorías" son unidades temáticas que permiten agrupar y organizar la información obtenida pudiendo ser de carácter teórico o empírico, y facilitan los procesos de interpretación, análisis y construcción de sentido (Rodríguez, 2005, como se citó en Suárez y Arenas, 2013). Las categorías en la TF son conceptos que representan fenómenos que agrupan aquellos acontecimientos, sucesos, objetos y acciones o interacciones que tienen una naturaleza similar

\footnotetext{
${ }^{1}$ La palabra experiencia se entiende como "experiencia vivida", es decir, "una forma de aprehensión del mundo, que contiene elementos emocionales que comprometen las subjetividades particulares. Tiene funciones prácticas en la vida cotidiana, refiriéndose al modo de existencia de los sujetos dentro de su realidad concreta y viviente" (Jodelet, 2006, p. 122).
} 
(Strauss y Corbin, 2002).

Se procedió a analizar las transcripciones por fases (Arenas-Monreal et al., 2013). En una primera fase se analizaron las entrevistas de forma individual por parte de los investigadores y se identificaron diversos factores que eran partícipes del proceso de inclusión del estudiantado (conceptos de discapacidad, inclusión escolar, apoyos pedagógicos, formación docente, saberes y conceptos compartidos, emociones que se desprenden de su quehacer docente, políticas públicas, grado de coherencia entre políticas públicas y discapacidad, necesidades administrativas, prácticas en el aula regular, apoyo de las familias de los estudiantes, actitudes de la sociedad en torno a la discapacidad, inserción laboral, semiología médica y psicológica entre otros).

Siguiendo la propuesta de Campo-Redondo y Labarca (2009) se realizó una selección inicial de categorías, las cuales pretendían encontrar relaciones de inclusión entre los factores presentes en las entrevistas. Luego se procedió a nombrar cada categoría etiquetando porciones de la información con un nombre que representó la interpretación de lo que pasaba en ese evento en particular -mencionado dentro de esa sección de la información.

En una segunda fase, Campo-Redondo y Labarca (2009) proponen la comparación de las diversas categorías, de manera que se clarifique lo que se percibe de la información, buscando las diferencias y similitudes entre las diversas categorías. De esa forma se dio una discusión entre los investigadores acerca de la codificación inicial para delimitar el alcance de cada categoría y su capacidad potencial para agrupar información. En esta segunda fase se realizó también un análisis de contenido para establecer si existía redundancia o falta de definición conceptual de las categorías iniciales y se construyó una serie de categorías emergentes, cada una con sus características y propiedades.

En una tercera fase se analizaron grupalmente las entrevistas para examinar la viabilidad de la codificación; para identificar las dimensiones de la información obtenida se introdujo el concepto de nodo temático, que daría cuenta de los postulados o improntas presentes en el imaginario social de los sujetos y que en cierta manera rigen sus sistemas de identificación (Moscovici y Vignaux, 2000). Las condiciones de saturación se dieron en esta fase, ya que se buscó agrupar los enunciados de las entrevistas en las categorías existentes para saber si éstas eran suficientemente robustas para contener lo dicho por los docentes o establecer si se necesitaban otras categorías. Se diferenció así cada entrevista para encontrar regularidades y diferencias y establecer las categorías y subcategorías finales (tablas I y II). Según Campo-Redondo y Labarca (2009) se verifican las categorías con la intención de identificar aquellas que sean más significativas.

Aumentando paulatinamente el nivel de generalidad del análisis, en una cuarta fase se abordó el análisis dinámico; en esta penúltima se elaboraron las tablas de concentración y matrices de datos a partir de la información encontrada en las entrevistas buscando sus relaciones dinámicas (figura 1). Según CampoRedondo y Labarca (2009) el agrupamiento de categorías en una forma teórica de análisis permite descubrir la llamada variable central, la cual explica el núcleo de sentido de las representaciones sociales.

Por último, el aspecto cuantitativo del análisis, más relacionado con el concepto de "emoción denotada" (Plantin, 2011), se llevó a cabo a través del software lexicométrico TXM² tratando de identificar la relevancia de algunos términos en función de su aparición recurrente.

\section{Resultados}

Se agruparon los contenidos de las entrevistas en categorías creadas a partir del análisis de los datos; es decir, categorías emergentes fueron identificadas como aglutinantes de las significaciones en torno a las dificultades inherentes al rol docente en la inclusión escolar (tabla I). Se identificó como categoría central

${ }^{2}$ TXM es un software de lexicometría desarrollado por el laboratorio ICAR (Interactions, Corpus, Apprentissage, Représentations - UMR 5191) y descargable desde http://textometrie.ens-lyon.fr/El análisis de concordancia permite identificar las ocurrencias de un término "pivote" y ordenarlo según sus contextos inmediatos (contexto izquierdo: hasta 10 palabras anteriores; el contexto derecho: hasta 10 palabras posteriores). 
la labor del docente como articulador de la norma sobre inclusión escolar y la realidad en la escuela.

Tabla I. Categorías emergentes sobre las dificultades inherentes al rol docente en la inclusión escolar

\begin{tabular}{|c|c|c|}
\hline & Categoría & Remite a \\
\hline 1 & $\begin{array}{l}\text { Dimensión profesional } \\
\text { e interdisciplinaria }\end{array}$ & $\begin{array}{l}\text { Remite a los conocimientos que tienen en tanto profesionales, lo que remite a sus } \\
\text { procesos formativos como docentes. Implica, además, las capacitaciones que les } \\
\text { ha brindado el Magisterio Nacional para abordar profesionalmente al } \\
\text { estudiantado en situación de discapacidad. }\end{array}$ \\
\hline 2 & Dimensión emocional & $\begin{array}{l}\text { Remite a las emociones que en ellos suscita el trabajo con el estudiantado en } \\
\text { situación de discapacidad y sus percepciones sobre el impacto que sobre las } \\
\text { familias tiene la atribución de discapacidad en sus hijos. }\end{array}$ \\
\hline 3 & Dimensión social & $\begin{array}{l}\text { Remite a la aceptación futura por parte de la sociedad del estudiantado en } \\
\text { situación de discapacidad; conlleva analizar la probabilidad de la incorporación de } \\
\text { los futuros ciudadanos en un marco laboral y sus dificultades cuando ya no tengan } \\
\text { una familia como red de apoyo. }\end{array}$ \\
\hline 4 & Dimensión legal & $\begin{array}{l}\text { Remite al marco legal en el cual se enmarca el trabajo en el aula con el } \\
\text { estudiantado en situación de discapacidad; así se discuten las normas legales y la } \\
\text { operacionalización de éstas en ambientes educativos concretos (sus escuelas). }\end{array}$ \\
\hline
\end{tabular}

La dimensión profesional y la dimensión legal se intersectan en las consecuencias de la implementación estatal a gran escala de la inclusión escolar de niños con discapacidad dentro de todas las aulas regulares en Colombia. Esta se ratifica con el decreto 366 de 2009 del MEN y se complementó posteriormente (MEN, 2012) intentando ofrecer una serie de orientaciones metodológicas para su implementación. Según los docentes, se vieron obligados a recibir y asumir los niños con discapacidad sin que para ello existiera una verdadera articulación entre lo que propone la norma y la realidad que se encuentra en la escuela, sin capacitación por parte de instituciones como la Secretaría de Educación del Distrito de Bogotá y la Secretaría de Educación Municipal de Cali. Los programas iniciales cursados para el ejercicio docente (normales superiores) no incluían módulos de formación en discapacidad o prácticas pedagógicas especializadas. Las consecuencias de ello se reflejan en las dimensiones emocional y social, las cuales se intersectan -pues instauran una preocupación por la demanda individual que genera esta población y la imposibilidad de asegurar un futuro personal y profesional de los estudiantes.

Se hallaron ocho subcategorías para reconstruir la teoría subjetiva de los docentes en torno a la inclusión educativa de población estudiantil en situación de discapacidad (tabla II): 
Tabla II. Subcategorías emergentes sobre la discapacidad y la inclusión escolar

\begin{tabular}{|c|c|c|}
\hline & Subcategoría & Remite a \\
\hline 1 & Atribución personal intrínseca & Ciegos, sordos, limitados físicos, enanos, niños especiales. \\
\hline 2 & Dificultad incapacitante & $\begin{array}{l}\text { Niños con problemas, incapaces, imposibilidad para hacer alguna cosa, } \\
\text { tener un problema específico, tiene necesidad de que lo traten } \\
\text { diferente, dificultad para aprender. }\end{array}$ \\
\hline 3 & Estado definido por la enfermedad & $\begin{array}{l}\text { Problema proveniente de una anomalía congénita o de un } \\
\text { traumatismo, enfermedad, trauma, problemas genéticos, esclerosis } \\
\text { múltiple, síndrome de Down, autismo. }\end{array}$ \\
\hline 4 & Forma de deficiencia & $\begin{array}{l}\text { Evoca alteraciones o pérdidas de las funciones o estructuras } \\
\text { corporales, sensoriales o cognitivas, deficiencia, déficit de atención, } \\
\text { deficiencia cognitiva, retardo mental, sordera, ceguera, deficiencia } \\
\text { cognitiva. }\end{array}$ \\
\hline 5 & $\begin{array}{l}\text { Forma de exclusión en las } \\
\text { actividades } \\
\text { de la vida cotidiana }\end{array}$ & $\begin{array}{l}\text { Ser excluido, no recibir atención, rechazado por los demás, estar } \\
\text { aislado. }\end{array}$ \\
\hline 6 & $\begin{array}{l}\text { Diferencias con las } \\
\text { representaciones sobre lo normal }\end{array}$ & $\begin{array}{l}\text { Dicotomía normal/anormal; evoca la idea de desviarse de las } \\
\text { representaciones colectivas que determinan lo que es admitido o } \\
\text { deseado. Es diferente, no es igual, tiene una diferencia. }\end{array}$ \\
\hline 7 & Emociones y valores & $\begin{array}{l}\text { Sentimientos, emociones y valores que generan o evocan las personas } \\
\text { que tienen una condición de discapacidad: afecto, solidaridad, valor, } \\
\text { angustia, fuerza, pesar, agotamiento. }\end{array}$ \\
\hline 8 & Necesidades de apoyo específicas & $\begin{array}{l}\text { Se evocan necesidades o estrategias de intervención apropiadas para } \\
\text { las personas en condición de discapacidad: necesitan ayuda específica, } \\
\text { necesitan de tratamiento especial, no se pueden tratar igual, } \\
\text { actitudes positivas, tenerle paciencia, darles apoyo. }\end{array}$ \\
\hline
\end{tabular}

Las primeras seis subcategorías remiten a una concepción de la discapacidad, especialmente en sus implicaciones pedagógicas, que está determinada por una concepción estática de la diferencia (Coll y Miras, 2001). Aquí, el diagnóstico clínico y sintomático de la "enfermedad" tiene un papel decisivo en términos de definir las necesidades específicas del individuo, a menudo ligadas a sus limitaciones sin tener en cuenta lo elementos del contexto. Lo anterior puede ocasionar algunas conductas de rechazo, tal como fue expresado por los docentes cuando hacen una diferenciación en función del tipo de discapacidad que tiene el niño. Las dos subcategorías restantes refieren a las necesidades específicas que se derivan del diagnóstico para un quehacer pedagógico y de las emociones que éste suscita.

Se identificó como categoría central en las Rs la labor del docente como articulador de la norma sobre inclusión escolar con la realidad de la escuela. En las prácticas educativas actuales convergen las estructuraciones, las políticas, los conceptos; para los docentes todo está a medio camino y -si bien se avanza en la inclusión del estudiantado en condición de discapacidad-el cómo implementar las políticas ha quedado bajo su tutela y liderazgo. El problema radica en que el sistema no contempla estrategias para prepararlos conceptual ni personalmente para asumir tal responsabilidad. Los docentes lo han expresado a través de sus relatos, el proceso inclusivo ha generado tensiones al interior de la escuela haciendo que dicho proceso sea difícil de afrontar.

Hernández (2014) propone un proceso de codificación abierta (a través de ella se intentan expresar los datos en forma de conceptos), de codificación axial (supone filtrar las categorías que han surgido en el paso anterior reorganizando la información y creando nuevas relaciones entre los conceptos) y la codificación selectiva (se selecciona una categoría central en torno a la que se organizan, se integran y se agrupa el resto de categorías). La figura 1 describe la teoría subjetiva de los docentes acerca del proceso de inclusión educativa de estudiantes en situación de discapacidad, resultante de la codificación axial y selectiva. 


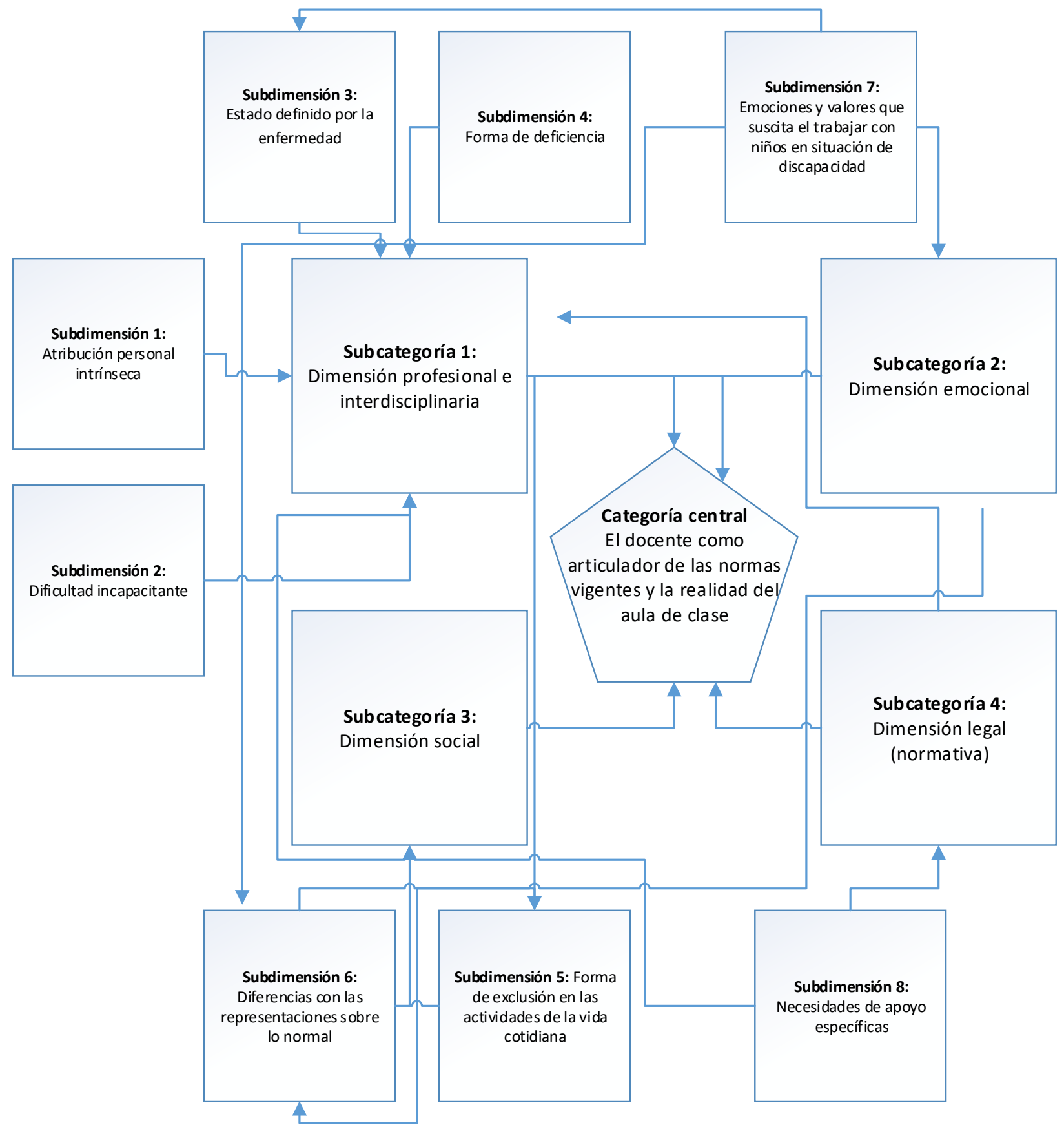

Fuente: Elaboración propia.

Figura 1. Integración dinámica sobre la inclusión del estudiantado en situación de discapacidad

Las relaciones dinámicas entre las subcategorías muestran que las problemáticas reales que afectan el proceso de inclusión en el aula tienen que ser resueltas por los docentes mismos, aunque sienten que esto excede sus capacidades personales, profesionales y organizacionales. Plantas físicas inadecuadas debido a la infraestructura de los colegios que carecen de rampas de acceso o baterías sanitarias especializadas, el estudiantado recibido en los cursos sin evaluaciones clínicas y diagnósticas que permitan entender la 
problemática individual de los estudiantes, contextos socioculturales desfavorecidos debido a la pobreza generalizada y bajo nivel académico de los padres que no les permite colaborar en los deberes académicos de sus hijos, la nula capacitación en discapacidad y pedagogía para docentes, la falta de apoyo interdisciplinario en sus prácticas cotidianas y la actitud del docente y la institución en general ante la discapacidad y la inclusión son factores que cada docente, de forma individual, debe afrontar si se desea proveer un trabajo pedagógico de calidad ante la inclusión escolar.

Esta articulación depende de los recursos de cada docente, para ellos no existe un apoyo tangible por parte del sistema educativo. La falta de articulación es puesta en evidencia, según ellos, a partir de la pobre movilización de recursos económicos y sociales con los que cuentan las instituciones, ejemplo de ello es la falta de materiales, la ambientación estética y física de los salones o la falta de personal de apoyo. La mayoría de los docentes entrevistados pone en duda la efectividad de la norma que lleva a la inclusión de niños en situación de discapacidad en el aula regular considerando que tal cambio produce más daño que beneficio a estos niños; algunos de ellos inclusive van a lamentar el cambio hacia este modelo educativo.

Respecto a la dimensión emocional se analizaron las emociones denotadas en el discurso que suscitan las vivencias de los profesores en las aulas regulares en su trabajo cotidiano con niños en condición de discapacidad y las dificultades que generó la implementación de los decretos sobre inclusión educativa. El repertorio de términos emocionales, inventariado a través del software TXM, se presenta en la tabla III.

Tabla III. Repertorio de términos emocionales

\begin{tabular}{lcc}
\hline \multicolumn{1}{c}{ Palabra } & Conteo & $\begin{array}{c}\text { \% Ponderado } \\
\mathrm{N}=24002\end{array}$ \\
\hline Difícil & 26 & 0.11 \\
\hline Tristeza & 16 & 0.07 \\
\hline Triste & 13 & 0.05 \\
Duro & 12 & 0.05 \\
Complicado & 11 & 0.05 \\
\hline Preocupado & 11 & 0.05 \\
\hline Preocupante & 10 & 0.04 \\
Frustrante & 8 & 0.03 \\
\hline Desesperado & 6 & 0.03 \\
Frustrado & 5 & 0.02 \\
Gratificante & 3 & 0.01 \\
Experiencia (linda) & 2 & 0.01 \\
\hline Experiencia (buena) & 2 & 0.01 \\
\hline
\end{tabular}

Como ha sido descrito por Plantin (2011) y Plantin y Gutiérrez (2011), la designación directa de la emoción es comprendida por la referencia que hace de un contexto específico. Se halla evidencia entonces para afirmar que la orientación emocional de las RS sobre la experiencia de la inclusión en el contexto educativo es intensa y orientada a lo negativo. La necesidad instaurada en los docentes de contar con evaluaciones previas (que permitan anticipar las fortalezas y debilidades de los estudiantes para crear adaptaciones curriculares de calidad) y la ausencia de capacitación son dos nodos temáticos relevantes para analizar las dificultades emocionales que conlleva la implementación cotidiana de los procesos inclusivos para los docentes participantes, quienes dejan entrever con claridad la necesidad del trabajo interdisciplinario como antesala del trabajo estrictamente pedagógico, buscando la aplicación de evaluaciones y diagnósticos específicos para cada niño con la esperanza de modificar los apoyos pedagógicos de manera eficiente y personalizada es lo que genera mayor dificultad para la implantación del modelo de educación inclusiva independiente de la ciudad (Bogotá y Cali).

Se analizó textométricamente el vocabulario emocional asociado a "discapacidad" buscando regularidades a través del software TXM, el resultado se presenta en la tabla IV, desglosado para Bogotá y Cali. 
Tabla IV. Concordancias para el término "discapacidad"

\begin{tabular}{|c|c|c|c|}
\hline Ciudad & Contexto izquierdo & Pivote & Contexto derecho \\
\hline $\mathrm{BOG}$ & trabajar con la & discapacidad & es difícil pero toca hacerlo \\
\hline BOG & $\begin{array}{l}\text { es muy difícil porque yo soy } \\
\text { consciente de todo el problema de la }\end{array}$ & discapacidad & \\
\hline BOG & trabajar con la & discapacidad & es difícil pero lo estoy haciendo \\
\hline BOG & $\begin{array}{l}\text { me da mucha tristeza porque no todo } \\
\text { el mundo se compromete con la }\end{array}$ & discapacidad & porque es difícil \\
\hline BOG & yo también tengo una personita con & discapacidad & y duele, duele muy fuerte, es muy duro \\
\hline BOG & $\begin{array}{l}\text { me da tristeza, me da mucha tristeza } \\
\text { porque no todo el mundo se } \\
\text { compromete con la }\end{array}$ & discapacidad & porque es difícil \\
\hline CALI & los niños con & discapacidad & $\begin{array}{l}\text { son muy difíciles, por ejemplo los niños } \\
\text { que tienen problemas de motricidad, los } \\
\text { niños con problemas de IMOC }\end{array}$ \\
\hline CALI & $\begin{array}{l}\text { es complicado, diría que difícil porque } \\
\text { hay niños con }\end{array}$ & discapacidad & que requieren de una educación especial \\
\hline
\end{tabular}

Pantiae (2017) ha puesto de manifiesto que ser exitoso en la implementación del proceso de la educación inclusiva está directamente relacionado con la acción docente en el aula y reconocer que la diversidad de necesidades contribuye a que diferentes formas de aprender sean reconocidas en el trabajo docente. Sin embargo, si la diversidad del aula se percibe como agotadora por parte del docente y con una carga emocional negativa, entonces el proceso de implementación puede afectarse significativamente. Hastings y Oakford (2003) afirman que la inclusión de niños con problemas emocionales y conductuales en espacios escolares tiene más probabilidades de tener un impacto negativo sobre otros niños, el maestro y el entorno escolar y del aula. Los resultados de esta investigación muestran que dicho impacto negativo es pronunciado en los docentes, no por la demanda generada en sí misma por la población estudiantil sino por la falta de apoyo de las instituciones a su labor docente.

\section{Discusión}

Las RS sobre la discapacidad dejan entrever que las personas siguen considerando que tener una discapacidad equivale a tener un déficit que afecta el funcionamiento de la persona y que es asimilable a estar enfermo; la discapacidad es entonces pensada en términos de un problema individual. Para López et al. (2010) es necesario reestructurar concepciones fuertemente arraigadas en la mente de profesores, especialistas y administradores educativos para poder disminuir la brecha entre los valores declarados en torno a la educación inclusiva y la realidad de las aulas.

Estas concepciones de los profesores dejan entrever que la realidad educativa de la inclusión se piensa desde una hegemonía médico-clínica que sobreestima el impacto de la necesidad que tienen los docentes de contar con apoyos terapéuticos específicos para el estudiantado en situación de discapacidad. La demanda constante de profesionales especializados, quienes serían las personas idóneas para dar respuesta a las problemáticas de los niños, deja al descubierto una ambivalencia entre la noción de ayuda y la comprensión de la diferencia, ¿cuáles serían entonces los tipos de ayuda que precisan los niños y que los docentes aún no están en capacidad de ofrecer? Una cosa es clara, en la medida que exista una consideración estática e individual de la discapacidad, la escuela continuará privilegiando el desarrollo de habilidades cognitivas en detrimento de concepciones más integrales sobre el aprendizaje (Escudero y Martínez, 2011).

Рara Lahlou y Abric (2011) los elementos constitutivos de una representación están jerarquizados, manteniendo entre éstos una relación que determina su significación y el lugar que ocupan dentro del sistema representacional. Teniendo en cuenta las Rs identificadas sobre la discapacidad y su núcleo central, los docentes serían los articuladores no preparados de la política pública y de la realidad del aula; este rol se les asignó sin capacitación alguna, por lo que la inclusión de estudiantes en situación de discapacidad a la escuela regular es vista de manera negativa, los docentes no asumen con entusiasmo el desafío de hacer de la escuela un lugar en el cual la diferencia sea considerada una condición aceptada y valorizada. 
Derivado del análisis de la emoción suscitada, y bajo el modelo de integración escolar imperante en Colombia, se identificó que para los docentes los niños con discapacidad son un reto abrumador. Para Paolini (2014) el estudio de las dinámicas emocionales de los docentes es de importancia, ya que éstas pueden promover o limitar los esfuerzos para construir el diseño de ambientes de aprendizaje. Dado que, desde un punto de vista emocional, las RS dan cuenta de una experiencia demandante y valorada mayoritariamente como negativa, es importante plantear opciones institucionales de formación en educación para la discapacidad, con el fin de lograr que los docentes cuenten con opciones válidas y relevantes en su quehacer profesional y acepten el desafío de la educación inclusiva.

El análisis desde la teoría fundamentada de las RS muestra que los docentes siguen considerando jerárquicamente la discapacidad como un problema individual y clínico, y que ellos supeditan sus acciones en el aula a la evaluación intuitiva de la discapacidad y sus consecuencias en el ámbito escolar. En este sentido, priman las explicaciones desde el modelo médico, al comprenderse la discapacidad como consecuencia de una enfermedad, sin tener en cuenta la influencia del contexto. De allí la importancia de las evaluaciones neuropsicológicas que tanto demandan los docentes.

Las RS sobre la discapacidad dejan entrever también que los docentes han actualizado sus conocimientos con relación a la discapacidad, apropiándose de nuevos términos y nuevas formas de "nombrar". De la Oliva et al. (2015) han propuesto cambiar el uso de términos como "discapacidad" por el de "estudiantes con dificultades para la formación", pues el uso de otros adjetivos para denominar a esta población sólo conlleva estigmatización. Todos los alumnos tienen "necesidades" y "dificultades para la formación", por lo tanto, no son conceptos estigmatizadores. Las Rs identificadas muestran que aún hay camino por recorrer en el complejo proceso de la inclusión escolar.

En el contexto educativo el concepto de inclusión escolar se ha asociado a la integración de estudiantes con necesidades educativas especiales al aula común, sin que esto signifique implementar cambios profundos en las prácticas y metodologías de enseñanza, ni mucho menos en la cultura de los centros escolares (Leiva, 2013). De tal forma, las RS identificadas en este estudio suponen un reto a la forma en que administrativamente se afronta la inclusión en los centros educativos, pues proponen la necesidad de implementar procesos formativos complementarios e interdisciplinarios para los docentes en torno a la inclusión escolar y a las implicaciones prácticas de trabajar con población con necesidades educativas especiales.

Fakolade et al. (2017) han afirmado que las actitudes hacia la inclusión son muy complejas y varían de maestro a maestro y de escuela a escuela. Las actitudes revelan que las maestras tienen una actitud más positiva hacia la inclusión de estudiantes con necesidades especiales que sus homólogos masculinos y que existe una diferencia significativa entre profesores casados y solteros en cuanto a los estudiantes con necesidades educativas especiales. En contraposición al trabajo de Fakolade et al. (2017), el análisis a partir de las RS muestra que, en el caso de la educación colombiana, en dos ciudades de dos regiones diferentes, no existen diferencias en torno a la forma en que se vivencia la inclusión de estudiantes con necesidades educativas especiales. No hay diferencias en los apoyos demandados, puesto que el sector educativo oficial en Colombia siempre ha carecido de recursos suficientes para proveer una educación de calidad (Cano y Serna, 2015), pero hay una convergencia hacia el espectro de lo negativo de las emociones que suscita la inclusión escolar.

Estudios como el de Ocete et al. (2015) revelan la importancia de trabajar las creencias que subyacen a las prácticas del profesorado, puesto que condicionan las actitudes facilitadoras u obstaculizadoras para la inclusión educativa. En la medida en que el sistema educativo integre propuestas interdisciplinarias efectivas para trabajar con los docentes en el ámbito de la educación inclusiva es que se podrá transformar el factor obstaculizador identificado en este estudio: el peso que recae en el docente de implementar políticas públicas en torno a la educación inclusiva y el peso emocional que esto genera.

Como conclusión preliminar podría decirse que las mejoras en el sistema educativo de cada país son una preocupación permanente en las agendas políticas de los gobiernos. La capacidad de brindar una educación pertinente y de calidad a todos los niños sin tener en cuenta sus características o sus condiciones de vida 
es uno de los mayores desafíos que toda sociedad debe afrontar. Puede decirse que, en las instituciones educativas públicas de Cali y Bogotá, la escolarización de niños en situación de discapacidad va por el camino de la inclusión o al menos es una preocupación manifiesta en toda la comunidad. Sin embargo, aún queda un vasto camino por recorrer, comenzando por las transformaciones culturales de los conceptos de inclusión y discapacidad. Estas transformaciones deben ser acompañadas indiscutiblemente de un proceso de formación, pues cuando los decretos y las estructuraciones se imponen sin el acompañamiento y las transformaciones necesarias, las secuelas sobre la vida escolar son considerables (Escudero y Martínez, 2011).

\section{Agradecimientos}

Este artículo tiene su origen en la investigación "Quel Sens pour l'Inclusion?", presentada por la autora para optar por el título de Master II recherche en Sciences de l'Éducation, Université Lumière (Lyon 2). El autor agradece a la Facultad de Psicología y a la Vicerrectoría de Investigaciones de la Pontificia Universidad Javeriana (Bogotá, Colombia) la financiación del proyecto "Representaciones Sociales sobre la Justicia" (ID SIAP 7800) que posibilitó su participación en esta investigación.

\section{Referencias}

Ainscow, M. (2012). Haciendo que las escuelas sean más inclusivas: lecciones a partir del análisis de la investigación internacional. Revista Educación Inclusiva, 5(1), 39-49.

https://www.revistaeducacioninclusiva.es/index.php/REl/article/view/220/214

Ainscow, M., Dyson, A., Goldrick, S. y West, M. (2013). Promoviendo la equidad en educación. Revista de Investigación en Educación, 11(3), 44-56. http://reined.webs.uvigo.es/index.php/reined/article/view/283

Arenas-Monreal, L., Ruiz-Rodríguez, M., Bonilla-Fernández, P., Valdez-Santiago, R. y Hernández-Tezoquipa, I. (2013). Cambios alimenticios en mujeres morelenses migrantes a Estados Unidos. Salud Pública de México, 55(1), 35-42. http://saludpublica.mx/index.php/spm/article/view/7186

Brito J. y Mansilla, J. (2013). Significados subjetivos de fortalezas y debilidades del proceso de inclusión educativa. Revista Latinoamericana de Educación Inclusiva, 7(2), 95-113.

http://repositoriocdpd.net:8080/handle/123456789/1869

Campo-Redondo, M. y Labarca, C. (2009). La teoría fundamentada en el estudio empírico de las representaciones sociales: un caso sobre el rol orientador del docente. Opción, 25(60), 41-54. https://www.redalyc.org/pdf/310/31012531004.pdf

Cano, M. V. A. y Serna, Y. Q. (2014). Las políticas educativas en Colombia y sus repercusiones en la formación y la enseñanza de las ciencias. Revista do Imea, 2(2), 47-59. https://revistas.unila.edu.br/IMEAUNILA/article/view/355/309

Coll, C. y Miras, M. (2001). Diferencias individuales y atención a la diversidad en el aprendizaje escolar. En A. Marchesi, C. Coll y J. Palacios (Comps.) Desarrollo Psicológico y Educación, Tomo 2 (pp. 331-353). Madrid: Alianza Editorial.

Cuevas, Y. (2015). Representaciones sociales de la reforma de educación básica: La visión de los directivos. Perfiles Educativos, 37(147), 67-85. https://doi.org/10.22201/iisue.24486167e.2015.147.47264

De la Oliva, D., Tobón, S., Pérez, A. K. y Romero, J. (2015). El proceso de inclusión social desde la socioformación: Análisis de concepciones sobre discapacidad y necesidades educativas especiales. Paradigma, 36(2), 49-73. http://revistaparadigma.online/ojs/index.php/paradigma/article/view/561/558

Escudero, J. M. y Martínez, B. (2011). Educación inclusiva y cambio escolar. Revista Iberoamericana de Educación, 55, 85-105. https://rieoei.org/historico/documentos/rie55a03.pdf 
España, A. E. y Gentiletti, M. G. (2011). Las representaciones sociales de los docentes de nivel medio sobre la enseñanza de la historia. Cuadernos de Educación, 9(9), 141-152.

https://revistas.unc.edu.ar/index.php/Cuadernos/article/view/828

Fakolade, O. A., Adeniyi, S. O. y Tella, A. (2017). Attitude of teachers towards the inclusion of special needs children in general education classroom: the case of teachers in some selected schools in Nigeria. International Electronic Journal of Elementary Education, 1(3), 155-169.

\section{https://www.iejee.com/index.php/IEJEE/article/view/272}

Garnique-Castro, F. y Gutiérrez-Vidrio, S. (2012). Educación básica e inclusión: un estudio de representaciones sociales. Magis, Revista Internacional de Investigación en Educación, 4(9), 577-593.

https://revistas.javeriana.edu.co/index.php/MAGIS/article/view/3577

Hassan, A. E. H., Alasmari, A. A. y Ehmed, E. Y. E. (2015). The influences of disabilities acceptance, knowledge about inclusive and interaction with disabilities peoples on trainee students' attitudes to educate intellectual disabilities in regular settings. International Journal for Innovation Education and Research, 3(10), 141-147. http://ijier.net/ijier/article/view/454/370

Hastings, R. P. y Oakford, S. (2003). Student teachers' attitudes towards the inclusion of children with special needs. Educational Psychology, 23, 87-94. https://doi.org/10.1080/01443410303223

Hernández, R. M. (2014). La investigación cualitativa a través de entrevistas: su análisis mediante la teoría fundamentada. Cuestiones Pedagógicas, 23, 187-210.

http://institucional.us.es/revistas/cuestiones/23/Mis 5.pdf

Jodelet, D. (1986). La representación social: Fenómenos, concepto y teoría. En Serge Moscovici, Pensamiento y vida social. Psicología social y problemas sociales (pp. 469-494). Paidós.

Jodelet, D. (2006). El otro, su construcción, su conocimiento. En S. Valencia (Coord.), Representaciones Sociales. Alteridad, epistemología y movimientos sociales. Universidad de Guadalajara/Maison des Sciences de L'Homme.

Lahlou, S. y Abric, J. C. (2011). What are the "elements" of a representation? Papers on Social Representations, 20, 1-20. http://eprints.lse.ac.uk/44355/

Leiva, J. J. (2013). De la integración a la inclusión: evolución y cambio en la mentalidad del alumnado universitario de educación especial en un contexto universitario español. Revista Electrónica Actualidades Investigativas en Educación, 13(3), 1-27. https://revistas.ucr.ac.cr/index.php/aie/article/view/12027

López, M., Echeíta, G. y Martín, E. (2010). Dilemas en los procesos de inclusión: explorando instrumentos para una comprensión de las concepciones educativas del profesorado. Revista Latinoamericana de Educación Inclusiva, 4(2), 155-176. http://www.rinace.net/rlei/numeros/vol4-num2/art8.pdf

Ministerio de Educación Nacional de Colombia. (2012). Orientaciones generales para la atención educativa de las poblaciones con discapacidad -PCD-en el marco del derecho a la educación. Imprenta Nacional.

Moscovici, S. (1961). La psychanalyse, son image et son public: Étude sur la représentation sociale de la psychanalyse [El psicoanálisis, su imagen y su audiencia: estudio sobre la representación social del psicoanálisis]. Presses Universitaires de France.

Moscovici, S. y Vignaux, G. (2000). The concept of Themata. En K. Duveen (Ed), Social Representations. Explorations in Social Psychology (pp. 156-183). Polity Press.

Ocete, C., Pérez-Tejero, J. y Coterón, J. (2015). Propuesta de un programa de intervención educativa para 
Facilitar la inclusión de alumnos con discapacidad en educación física. Retos: Nuevas Tendencias en Educación Física, Deporte y Recreación, 27, 140-145. https://recyt.fecyt.es/index.php/retos/article/view/34366/18545 
Pantiae, N. (2017). An exploratory study of teacher agency for social justice. Teaching and Teacher Education, 66, 219-230. https://doi.org/10.1016/j.tate.2017.04.008

Paolini, P. V. R. (2014). Emociones en contextos académicos. Perspectivas teóricas e implicaciones para la práctica educativa en la universidad. Electronic Journal of Research in Educational Psychology, 12(3), 567-596. http://www.investigacion-psicopedagogica.org/revista/new/ContadorArticulo.php?959

Plantin, C. (2011). Les bonnes raisons des émotions: Principes et méthode pour l'étude du discours «émotionné» [Buenas razones para las emociones: principios y método para el estudio del "discurso emocional]. Peter Lang.

Plantin, C. y Gutiérrez, S. (2011). Argumentar por medio de las emociones: La "campaña del miedo" del 2006. Estudios de Comunicación y Política, 24, 41-69. https://hal.archives-ouvertes.fr/halshs-00853888/

Strauss, A. y Corbin, J. (2002). Bases de la investigación cualitativa, técnicas y procedimientos para desarrollar la teoría fundamentada. Editorial Universidad de Antioquia.

Suárez, E. y Arenas, J. (2013). La saturación teórica en la teoría fundamentada: su de-limitación en el análisis de trayectorias de vida de víctimas del desplazamiento forzado en Colombia. Revista Colombiana de Sociología, 36(2), 93-114. https://revistas.unal.edu.co/index.php/recs/article/view/41641/43310

Thaver, T., Lim, L. y Liau, A. (2014). Teacher variables as predictors of Singaporean pre-service teachers' attitudes toward inclusive education. European Journal of Research on Social Studies, 1(1), 1-8. http://included.eu/sites/default/files/documents/e010101.pdf

UNESCO (1994). World conference on special needs education: Access and quality. Salamanca, Spain 7-10 June, 1994. The Salamanca Statement and Framework for Action on Special needs Education. Paris (ED94/WS/18). https://unesdoc.unesco.org/ark:/48223/pf0000098427

Yarza, A. (2010). Del destierro, el encierro y el aislamiento a la educación y la pedagogía de anormales en Bogotá y Antioquia. Principios del siglo XIX a mediados del siglo XX. Revista Educación y Pedagogía, 22(57), 111-129. http://aprendeenlinea.udea.edu.co/revistas/index.php/revistaeyp/article/view/7364

Yarza, A., Ramírez, M., Franco, L. M. y Vásquez, N. (2013). Historia polifónica de la escolarización/educación para personas con discapacidad(es) desde las voces de educadores especiales en Medellín (Antioquia, Colombia): 1965-2002. Revista Temas de Educación, 19(2), 83-105.

https://revistas.userena.cl/index.php/teduacion/article/view/449 